\title{
Systemic analysis of IncRNA and miRNA expression profiles with associated ceRNA network in glioblastoma
}

\author{
Hu Sun ${ }^{1,2}$, Xinhua Liu ${ }^{3}$, Wei Song ${ }^{4}$ and Yugong Feng ${ }^{1}$ \\ ${ }^{1}$ Department of Neurosurgery, The Affiliated Hospital of Qingdao University, Qingdao, Shandong, 266003, China \\ ${ }^{2}$ Department of Neurosurgery, Zibo Central Hospital, Zibo, Shandong, 255036, China \\ ${ }^{3}$ School of Biomedical Engineering, Tianjin Medical University, Tianjin, 300070, China \\ ${ }^{4}$ Health examination center, Zibo Central Hospital, Zibo, Shandong, 255036, China
}

Correspondence to: Yugong Feng, email: yugongfeng@yeah.net

Keywords: ceRNA network; glioblastoma; InCRNA; TCGA

Received: June 28, 2017

Accepted: December 01, 2017

Published: January 10, 2018

Copyright: Sun et al. This is an open-access article distributed under the terms of the Creative Commons Attribution License 3.0 (CC BY 3.0), which permits unrestricted use, distribution, and reproduction in any medium, provided the original author and source are credited.

\section{ABSTRACT}

Glioblastoma (GBM) is the most common malignant brain tumor with high morbidity and mortality. Long and small non-coding RNA, i.e. IncRNA and miRNA in this study, play important roles in progression of complex diseases and competes with each other when regulate expression of mRNA. In this study, we conducted systemic analysis of high-throughput gene sequencing and microarray datasets to explore expression regulation patterns in GBM. Differential expression mRNAs, IncRNAs and miRNAs were obtained through comprehensively comparinged their expression profiles between GBM and adjacent non-tumor tissues. As a result, a total of 28 IncRNAs were found to be aberrantly expressed ( $\mid \log 2$ (fold change)| > 1 , corrected $p$-value $<0.01$ ) and six out of them were significantly associated with GBM prognosis. The expression changes of the six IncRNAs were further validated through quantative PCR analysis. Finally, IncRNA mediated competition endogenous (ceRNA) network was constructed, which should be helpful for the understanding of IncRNA mediated ceRNA network and identification of prognosis biomarker for GBM.

\section{INTRODUCTION}

Despite grave progress of glioblastoma (GBM) care and treatment, its prognosis is still poor and large variability in patients' outcome is observed. In United States, the overall survival of untreated GBM diagnosed from 2000-2003 was only 8.1 months and it was still lower than one year even if take those underwent surgery and radiation into account [1]. What's more, lots of novel methods only benefit for specific patients, such as the adjuvant temozolomide with radiotherapy could only affect GBM patients with specific MGMT methylation status compared with those radiotherapy only [2]. It has been widely accepted that GBM is a genetic-driven disease and influenced by variations of multi genes [3]. While, its mechanisms are still largely unknown. In this case, it is urgent to identify potential prognosis biomarkers for GBM.

Long non-coding RNAs (lncRNAs) are non-coding RNA first described in high-throughput sequencing of full- length cDNA [4] with length longer than 200 nucleotides. They were taken as transcriptional noise for their lacking of translation potential. Recent studies have revealed their roles in carcinogenesis and cancer metastasis $[5,6]$ and more and more attentions have been paid on them. One of the most attractive and enigmatic points is how lncRNAs regulate gene expression. The most accepted mechanism of lncRNAs' gene regulation function is chromatin modification, i.e. mediates epigenetic changes by recruiting chromatin remodeling complexes to specific genomic loci [7]. Besides, lncRNAs could act as microRNA (miRNA) sponge to reduce miRNA regulatory effect on mRNA [8], and thus up-regulate expression of miRNA target genes. Although the major mechanism of lncRNAs is the regulation of expression of neighboring genes, lncRNAs could also serve as scaffold for proteinprotein interactions or decoys to proteins [9]. Additionally, lncRNAs can regulate kinase functions. LncRNA NBR2 engages a metabolic checkpoint by regulating AMP- 
activated protein kinase (AMPK) under energy stress [10]. Accumulating evidence suggests that lncRNAs play a role in fundamental biological functions, and dysregulation of lncRNAs contributes to cancer development, progression, and metastasis in many malignancies [11]. MiRNA, another type of well-studied non-coding RNA, could repress gene expression through binding complementary sequences in mRNAs, which known as miRNA response elements (MRE) [12]. A lot of microRNA signatures have been reported and there is an increasing possibility of using microRNAs as novel biomarkers and tools of treatment for tumor [13]. Previous studies have proposed and validated the hypothesis of competing endogenous RNA (ceRNA), i.e. IncRNAs, mRNAs and other types of RNAs act as miRNA sponges to suppress the function of miRNAs by using shared MRE $[14,15]$. So, lncRNA could repress the function of miRNA, and miRNA could affect mRNA expression. And thus, the three types of RNA form the IncRNA-miRNA-m RNA ceRNA network, which should play important roles in many types of diseases. Liang and collages [16] illustrated that lncRNA H19 promotes epithelial to mesenchymal (ETM) transition by acting as miRNAs sponge in colorectal cancer. Endogenous miRNA sponge lincRNA-RoR regulates Oct4, Nanog, and Sox 2 in human embryonic stem cell self-renewal [17]. Zhang et al constructed lncRNA-miRNA-mRNA interaction network to explore breast cancer progression based on bioinformatics analysis [18]. So, we believe that exploration of interaction between lncRNAs, miRNAs, and mRNAs should be helpful for the identification of prognosis biomarkers for cancer.

In this study, we conducted systemic analysis of high-throughput RNA sequencing and microarray datasets of GBM. Differential expression lncRNAs (DEL), miRNAs (DEMI), and mRNAs (DEM) were identified in 156 GBM samples compared with adjacent normal tissues. As a result, 28 DELs, 47 DEMIs, and 3,329 DEMs were identified. Eventually, 1 miRNA, 28 lncRNAs, and 2 mRNAs were used for the construction of lncRNA-miRNA-mRNA ceRNA network and 6 lncRNAs were found to be significantly associated with prognosis of GBM. Our study should be helpful for the understanding of IncRNA-miRNA-mRNA regulation patterns and the therapy of GBM.

\section{RESULTS}

\section{Differential expression mRNAs}

Principle component analysis (PCA) through prcomp command in $\mathrm{R}$ obtained two main sample clusters, i.e. GBM samples and adjacent normal brain samples (Figure 1A). Consistent with PCA result, there are 3357 DEM (1,489 (44.36\%) down-regulated and 1,816 (55.64\%) up-regulated genes) were identified in PG compared with NG samples, while, only 124 mRNAs (21 down-regulated (16.94\%) and 103 (83.06\%) up-regulated genes) were found to be differential expression in $\mathrm{RG}$ compared with PG samples. Figure $2 \mathrm{~B}$ and $2 \mathrm{C}$ illustrated read count ( $\log 2$ scale) mapped to each gene in $P G$ samples versus NG samples and RG samples versus PG samples respectively.

\section{Enriched KEGG pathways}

Figure 2A left and 2B left show the heatmap of top 100 down-regulated and 100 up-regulated DEM in PG and all of the DEM in RG. A total of 30 and 5 KEGG pathways were found to be significantly enriched in DEM of PG and RG. Figure $2 \mathrm{~A}$ right and $2 \mathrm{~B}$ right illustrate the pathway map of the most significantly enriched pathway of PG and $\mathrm{RG}$ respectively visualized by pathview package [26]. The top 10 most significantly enriched pathways of PG are listed in Table 1.

\section{Differential expression IncRNAs}

Based on the criteria of $\mid \log 2$ (fold change) $\mid>1$ and FDR adjusted $p$-value $<0.05$, a total of $28 \operatorname{lncRNAs}$ (Table 2) that with annotation in GENCODE IncRNA version26 were found to be differential expression in PG compared with $\mathrm{NG}$. While, there is no DEL in RG samples compared with PG samples. So, the following analysis are mainly focused on primary GBM and adjacent normal brain samples.

\section{Differential expression miRNAs}

A total of 47 miRNAs (31 (65.96\%) down-regulated and 16 (34.04) up-regulated genes) were found to be significantly differential expression in GBM samples compared with adjacent normal brain samples. Figure 3A illustrate miRNA levels in GBM samples versus adjacent normal brain tissues. Figure 3B is the two-way cluster of DEMI and samples visualized by pheatmap package. As our expected, two main clusters, i.e. GBM and normal brain tissues clusters, were obtained.

\section{ceRNA network}

Through miRcode, we obtained 73 lncRNA-miRNA interaction pairs. While, there are only two miRNAmRNA regulation pairs in miRTarBase, i.e. miR-139-5PCXCR4 and miR-139-5P-NR5A2, that were supported by at least two of the following evidences, i.e. qRT-PCR, western blot, microarray and ChIP-seq, luciferase reporter assay. Strikingly, miR-139-5P were found to be regulated by all of the 28 DEL. The ceRNA network is shown in Figure 4A. Survival analysis based on survival package of R identified 6 lncRNAs, including RP11-273G15.2 $(p$-value $=0.00013), \mathrm{RP3}-439 \mathrm{~F} 8.1(p$-value $=0.027)$, RP11-286B14.1 ( $p$-value $=0.037), \mathrm{RP} 11-161 \mathrm{H} 23.9$ $(p$-value $=0.039), \operatorname{LINC01579}(p$-value $=0.046)$ and 
Table 1: The top 10 most significantly enriched KEGG pathways of PG

\begin{tabular}{lllc}
\hline Pathway ID & Pathway Name & $\boldsymbol{P}$-value & Gene Count \\
\hline hsa04020 & Calcium signaling pathway & $3.74 \times 10^{-15}$ & 58 \\
hsa04724 & Glutamatergic synapse & $1.23 \times 10^{-14}$ & 44 \\
hsa04723 & Retrograde endocannabinoid signaling & $4.90 \times 10^{-13}$ & 39 \\
hsa04727 & GABAergic synapse & $1.08 \times 10^{-12}$ & 35 \\
hsa04080 & Neuroactive ligand-receptor interaction & $1.00 \times 10^{-11}$ & 69 \\
hsa05032 & Morphine addiction & $5.54 \times 10^{-11}$ & 34 \\
hsa04713 & Circadian entrainment & $2.09 \times 10^{-10}$ & 34 \\
hsa04921 & Oxytocin signaling pathway & $2.70 \times 10^{-9}$ & 44 \\
hsa04725 & Cholinergic synapse & $1.87 \times 10^{-8}$ & 34 \\
hsa04720 & Long-term potentiation & $2.26 \times 10^{-8}$ & 25 \\
\hline
\end{tabular}

LY86-AS1 ( $p$-value $=0.049)$, that significantly associated with the overall survival of GBM and they might provide valuable prognosis biomarkers for GBM.

Besides, to obtain more comprehensive miRNAmRNA regulation patterns in GBM, we searched miRNAmRNA pairs from TargetScan database containing DEMI and DEM. As a result, we obtained a total of 590 miRNAmRNA regulation pairs containing 11 DEMIs and 442
DEMs. The full list of miRNA-mRNA regulation pairs were provided in Supplementary Table 1.

\section{qPCR analysis}

For lncRNAs significantly associated with GBM survival, we further validated their expression changes in U251 and normal cell lines through qPCR analysis. As a

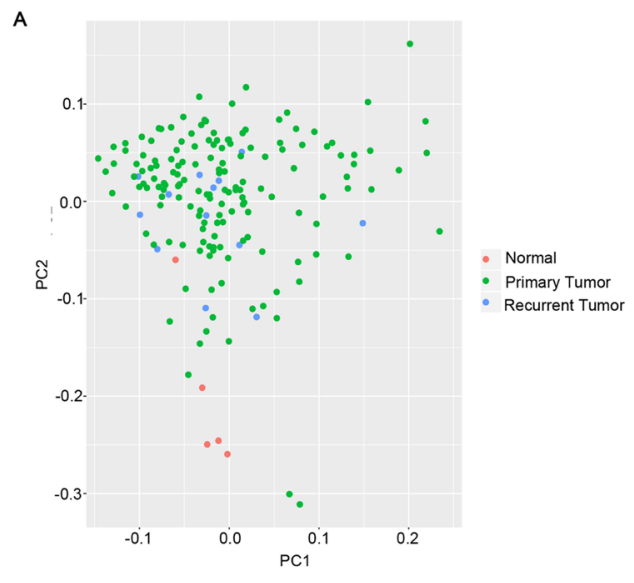

B

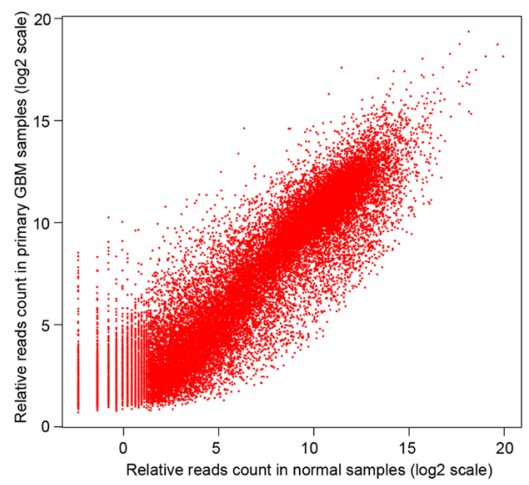

C

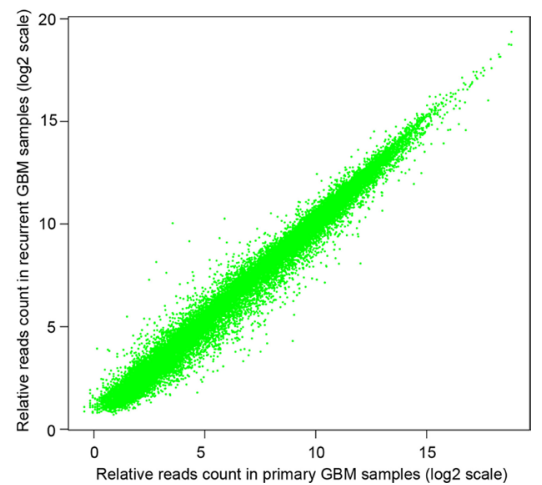

Figure 1: Analysis overview of RNA expression profile from TCGA. (A). Principle component analysis (PCA) of GBM and adjacent normal brain tissue samples. $(\mathbf{B})$ and $(\mathbf{C})$ is the scatter plot of relative read count $(\log 2$ scale) mapped to every gene in PG versus NG and RG versus PG respectively. 
Table 2: DEL in PG samples compared with NG samples

\begin{tabular}{lccccc}
\hline Gene ID & Log2(fold change) & FDR & Gene ID & $\begin{array}{c}\text { Log2(fold } \\
\text { change) }\end{array}$ & FDR \\
\hline ENSG00000258754 & 4.59 & $8.63 \times 10^{-19}$ & ENSG00000255176 & -3.03 & $1.26 \times 10^{-8}$ \\
ENSG00000257151 & -3.52 & $6.78 \times 10^{-16}$ & ENSG00000246323 & -2.10 & $6.01 \times 10^{-8}$ \\
ENSG00000253552 & 7.57 & $8.20 \times 10^{-16}$ & ENSG00000237424 & 3.21 & $7.13 \times 10^{-8}$ \\
ENSG00000249859 & 2.23 & $2.27 \times 10^{-11}$ & ENSG00000177133 & -2.94 & $1.61 \times 10^{-7}$ \\
ENSG00000145063 & -4.18 & $4.59 \times 10^{-11}$ & ENSG00000216863 & -4.48 & $1.77 \times 10^{-7}$ \\
ENSG00000233452 & -2.76 & $2.94 \times 10^{-10}$ & ENSG00000242512 & 4.01 & $2.55 \times 10^{-7}$ \\
ENSG00000233705 & -4.88 & $5.47 \times 10^{-10}$ & ENSG00000215196 & -4.02 & $4.76 \times 10^{-7}$ \\
ENSG00000258334 & 4.10 & $6.85 \times 10^{-10}$ & ENSG00000259834 & -3.09 & $9.95 \times 10^{-7}$ \\
ENSG00000246363 & -3.03 & $9.42 \times 10^{-10}$ & ENSG00000259583 & -3.31 & $3.61 \times 10^{-6}$ \\
ENSG00000259326 & -2.37 & $1.40 \times 10^{-9}$ & ENSG00000253553 & -2.09 & $3.73 \times 10^{-6}$ \\
ENSG00000204261 & 2.32 & $1.53 \times 10^{-9}$ & ENSG00000228971 & -4.48 & $4.71 \times 10^{-6}$ \\
ENSG00000249001 & 6.33 & $2.06 \times 10^{-9}$ & ENSG00000213468 & 2.02 & $8.95 \times 10^{-6}$ \\
ENSG00000256982 & -3.99 & $2.11 \times 10^{-9}$ & ENSG00000247317 & -2.39 & $9.25 \times 10^{-6}$ \\
ENSG00000259462 & -2.88 & $4.75 \times 10^{-9}$ & ENSG00000234869 & 3.51 & $9.33 \times 10^{-6}$ \\
\hline
\end{tabular}
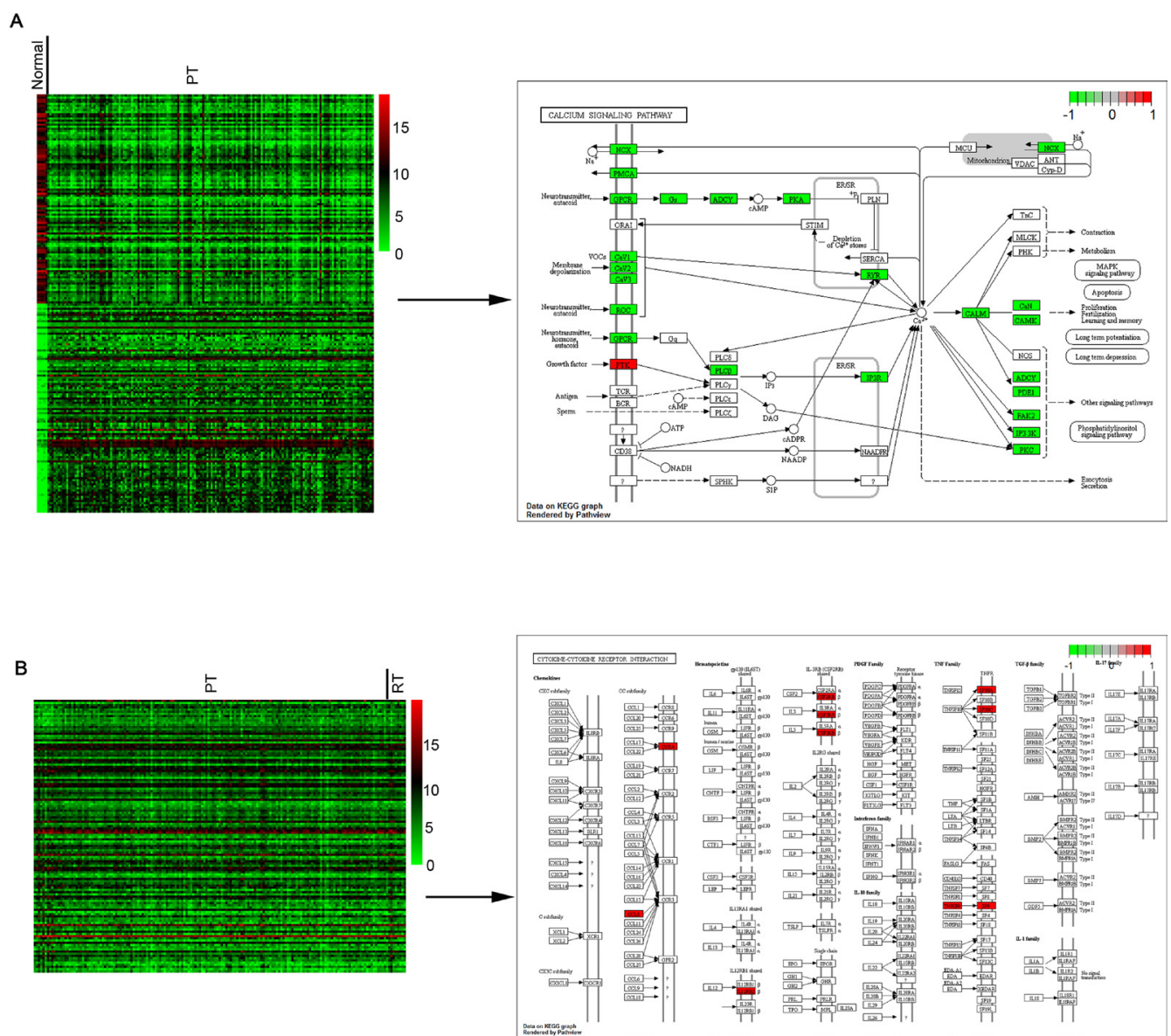

Figure 2: KEGG pathway analysis of DEM in PG and RG. (A). Heatmap of the top 100 down-regulated and 100 up-regulated genes in PG samples (left). The right panel represents the most significant KEGG pathway of PG DEM. (B). Heatmap of DEM in RG samples (left). The right panel represents the most significant KEGG pathway of RG DEM. Green, red and white box in right panel of (A) and (B) represents down-regulated, up-regulated and non-differential expression genes respectively. 
A

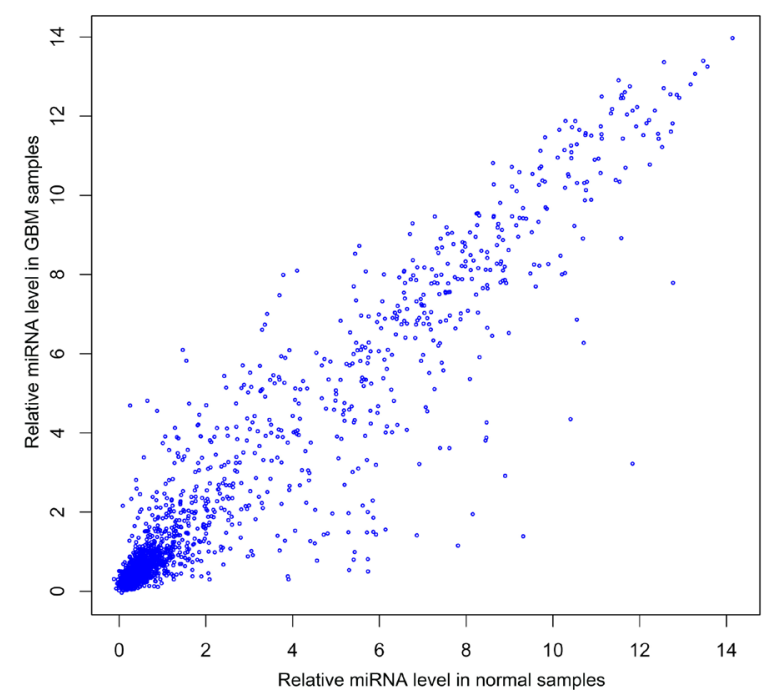

B

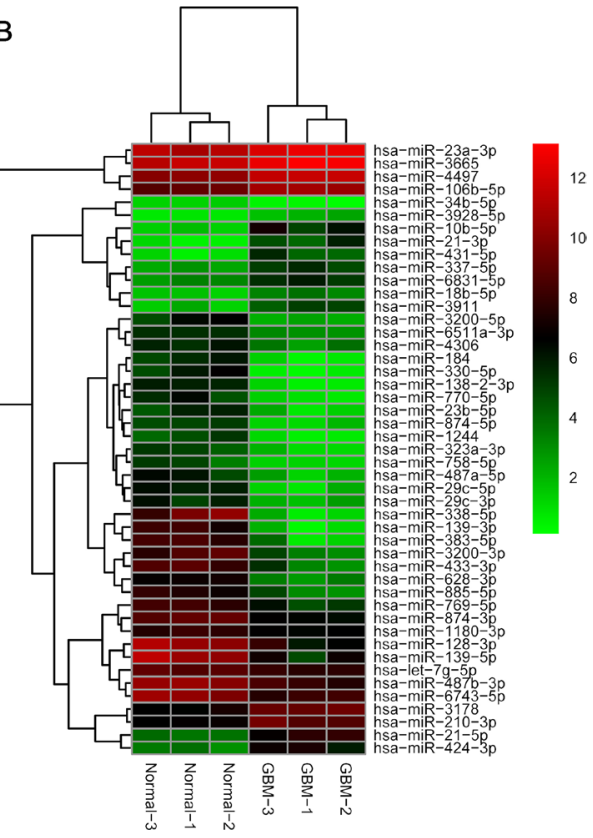

Figure 3: Analysis overview of miRNA expression profiles. (A). Scatter plot of relative miRNA level (log2 scale) of GBM sample versus adjacent normal brain tissues. (B). Two-way cluster of DEMI and samples. X-axis and Y-axis represents DEMI and samples respectively.

A

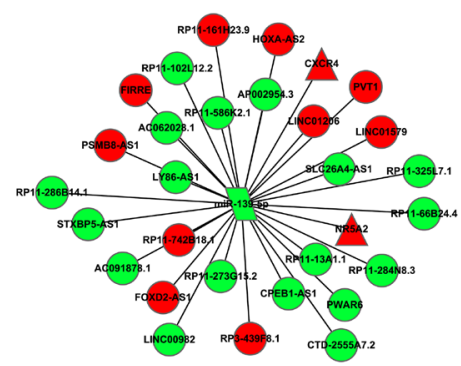

B
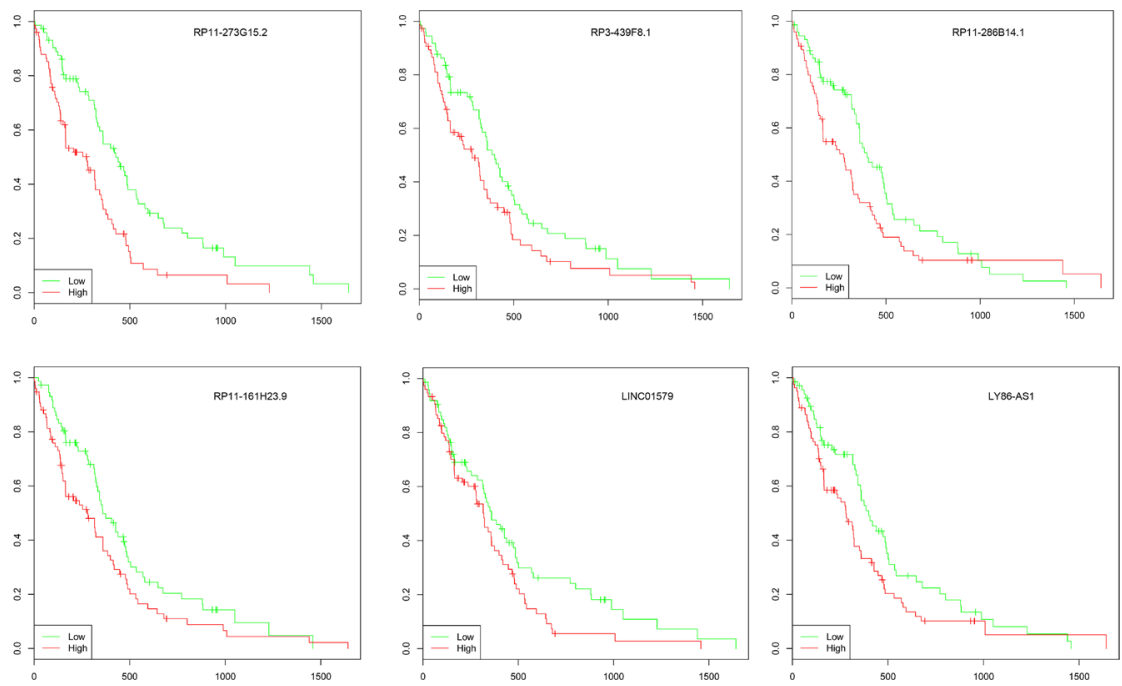

Figure 4: CeRNA network and survival analysis. (A). CeRNA network. Diamonds, circle and triangles represent lncRNAs, miRNA and mRNAs respectively. Green and red color is down-regulated and up-regulated genes. (B). Kaplan meier plots of six lncRNAs that significantly associated with the survival of GBM. Green and red line is the GBM samples with lower and higher (based on the median of all GBM expression values) gene expression values respectively. 
result, the results of all of the six lncRNAs were consistent with the microarray analysis, i.e. significantly up-regulated in GBM cell lines compared with normal cells (Figure 5).

\section{DISCUSSION}

More and more evidences indicate that lncRNAs play important roles in the progression of human cancer. Several studies have shown the association between aberrant lncRNA expression and different types of cancers $[27,28]$. In recent years, some efforts have been made to uncover dysregulated lncRNAs in GBM through gene microarray or RNA-sequencing [29-31]. However, no study has focused on the ceRNA network which reflecting lncRNA-miRNA-mRNA interaction or regulation pairs for GBM.

In previous studies, lncRNAs were considered as junk RNAs for their lack of translation potential. While, recently studies identified several lncRNAs as valuable diagnosis and prognosis biomarkers in cancers and other
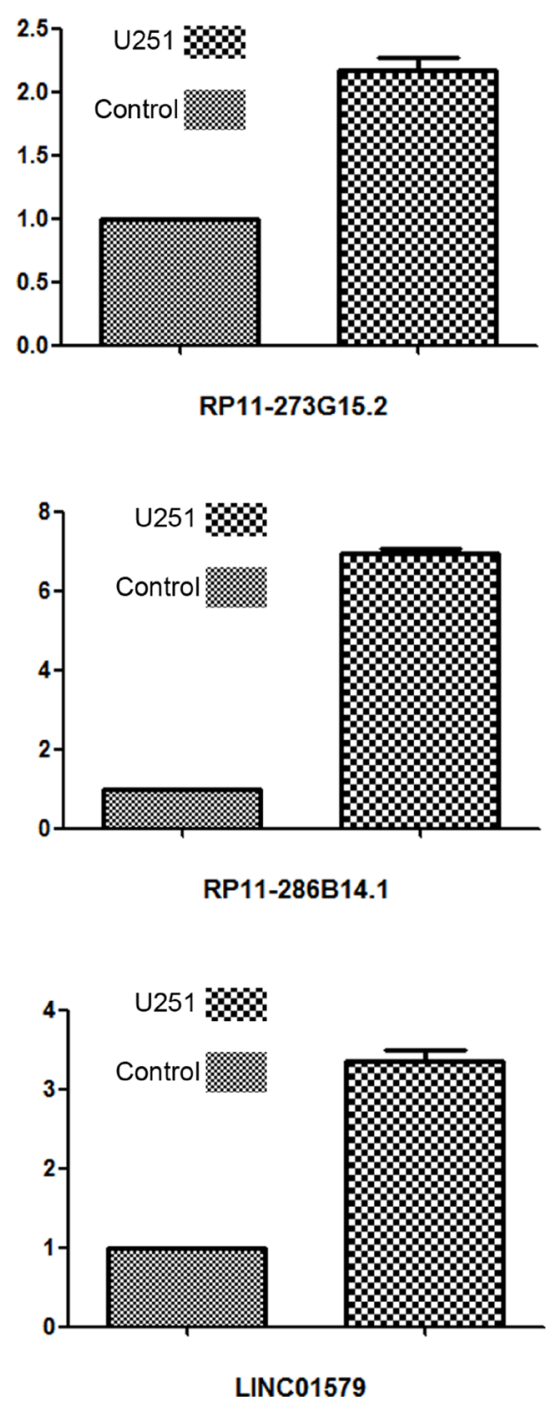

types of complex diseases, such as lncRNA-p53, H19, MALAT1 [16, 32, 33]. In this study, besides differential expression analysis of IncRNA, miRNA and mRNA, we also drew our attention to the prognosis significance of DEL. As a result, 6 out of the 28 DEL were found to be significantly associated with the survival of GBM, i.e. RP11-273G15.2, RP3-439F8.1, RP11-286B14.1, RP11161H23.9, LINC01579 and LY86-AS1. As to LY86-AS1, previous studies have proved its potential in several types of cancer, including myeloid cancer, colorectal cancer [34-36]. Our study confirmed the novel function of LY86-AS1 in GBM. While, the other 5 DEL were firstly determined as cancer prognosis biomarkers, and further studies are still needed to confirm their roles in GBM.

MiRNA plays an important role in tumor immune response. For example, miR-9 is overexpressed in several types of malignancies, including lung cancer, which resulting in down-regulation of MHC class I and preventing cancer cells detection through the immune system [37]. MiR-139-5p is the only miRNA in ceRNA
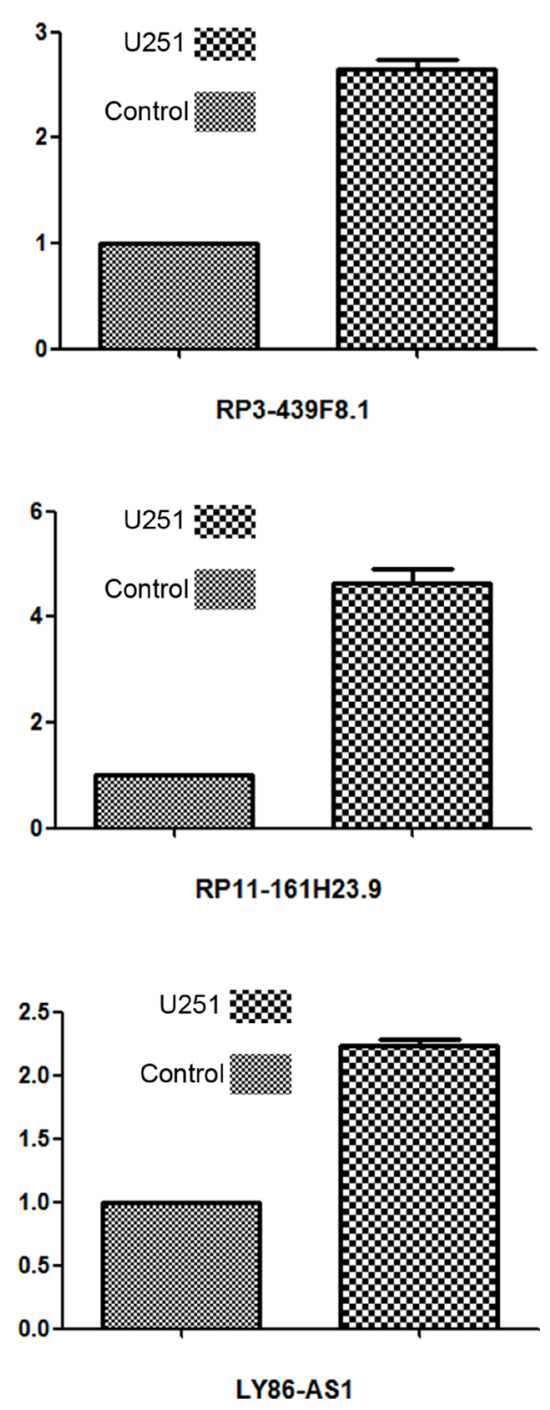

Figure 5: qPCR analysis of the six IncRNAs significantly associated with GBM survival. 
network, which have been proved to associated with the progression of multi human cancers, such as breast cancer, liver cancer, as well as esophageal cancer [3840]. Dysregulated calcium signaling is a classical cancer hallmark and provides chance for cancer diagnosis and therapy [41-43]. Calcium signaling pathway is the most significant pathway of DEM in PG compared with adjacent normal brain tissues and miR-139-5p was proved to be associated calcium metabolism by previous studies $[38,44,45]$. Consistent with our study, several molecular biology and bioinformatics analysis also proved the association between miR-139-5p and GBM [46-48].

CXCR4 and NR5A2 are the two DEM that regulated by miR-139-5p in ceRNA network. CXCR4 and NR5A2 are all reported to be associated with the progression of human cancers [49-52]. Besides, CXCR4 has been proved to be related to GBM $[53,54]$. As to NR5A2, it encodes a DNA-binding zinc finger transcription factor protein and involved in the expression of genes for hepatitis B virus and cholesterol biosynthesis. It inhibit pancreatic cancer stem cell properties [55] and associated with pancreatic cancer risk [56]. While, no study has focused on its role in GBM, our study should provide novel evidence of potential of NR5A2 on the survival of GBM.

In conclusions, our study presents overall ceRNA network pattern in GBM, and provides several known and novel diagnosis and prognosis biomarkers. This should be helpful for the treatment of GBM.

\section{MATERIALS AND METHODS}

\section{TCGA and GEO datasets}

RNA sequencing datasets were obtained from TCGA which contained 156 primary GBM samples, 13 recurrent GBM samples and 5 adjacent normal brain tissues. MiRNA expression profiles were downloaded from GEO with the accession number of GSE65626. A total of 3 GBM and 3 adjacent normal brain tissues were used for the total RNA extraction and miRNA/mRNA profiling based on commercial affymetrix mRNA and miRNA microarray platform.

\section{Differential expression analysis}

The RNA sequencing data were classified into 3 cohorts, i.e. primary GBM (PG), recurrent GBM (RG) and adjacent normal brain tissues (NG). The single expression data was combined and filtered RNA and miRNA data with no expression values. The raw count data were normalized based on DESeq version2 [19], a free available R Bionconductor package, and differential expression RNAs in PG compared with NG, and in RG compared with PG, were identified using DESeq command by instructions of DESeq manual. MiRNA microarray datasets were firstly preprocessed by affy package [20] of R, mainly including background correction and normalization. Linear Models for Microarray and RNA data (limma) package [21] was used for the identification of DEMI in GBM samples compared with adjacent normal brain tissues. Genes with absolute fold change ( $\log 2$ scale) $>1$ and FDR adjusted $p$-value $<0.05$ were considered as significant.

\section{Pathway enrichment analysis}

To uncover the biological processes involved in the progression of GBM, we conducted functional enrichment analysis for DEM in PG and RG in the Database for Annotation, Visualization and Integrated Discovery (DAVID) [22]. KEGG pathway with $p$-value $<0.05$ were considered as significantly enriched in DEM.

\section{Identification of differential expression IncRNAs}

To obtain DEL from the total differential expression RNAs, we intersected the GENCODE lncRNA annotation version26 [23] with our result. Non-coding RNAs not included in the annotation were excluded.

\section{Construction of ceRNA network}

LncRNA-miRNA interactions and miRNA targeted mRNAs were obtained through miRcode [24] and miRTarBase [25] respectively. For miRNA-mRNA pairs, only those validated by at least two of the following methods, i.e. qRT-PCR, western blot, microarray and ChIP-seq, luciferase reporter assay, were retained.

To obtain more comprehensive landscape of miRNA-mRNA regulation pairs, we further explore miRNA-mRNA pairs from TargetScan database (http:// www.targetscan.org/vert_71/). The miRNA-mRNA network containing DEMI and DEM was obtained. Supplementary Figure 1 illustrates the distribution of miRNA-mRNA regulation number that validated by different experimental methods number.

\section{qPCR analysis}

For the identified therapeutic targets, we further validated their expression changes through qPCR analysis in U251 and normal cells. The human U251 cell line was purchased from ATCC (American Type Culture Collection, Nr. TIB-202, Wesel, Germany) and was cultured in RPMI1640 media (Sigma-Aldrich) supplemented with 10\% FBS (PAA), 20mMHEPES (Sigma-Aldrich). Cells were maintained at densities between 0.2 and $1.0 \times 10^{6}$ cells $/ \mathrm{ml}$ in culture. Cells were plated in $24-w e l l$ plates $(3 \times 105$ cells/ well) and treated with $10 \mathrm{ng} / \mathrm{ml} \mathrm{IL-1betab}$ (Invitrogen, Carlsbad, CA, USA) for 24 h. Primers: RP11-273G15.2p5: ATCTTTTCCACCTTCCCAATCCT, RP11-273G15.2p3: GGCTTGTGAAGGGCTTTCGT; RP3-439F8.1p5: TAA AGTTGTGCGCTGAGCATC, RP3-439F8.1p3: GGTC 
CAGCATTGTCGTCTGA; RP11-286B14.1p5: CCGC AGCTACGAAAAACCAT, RP11-286B14.1p3: GGGTCA CTCACTCATGCGAA; RP11-161H23.9p5: GCAGT GAGCTGGACAAGTCT, RP11-161H23.9p3: ATGCCA AGAGAGTGACAGCC; LINC01579p5: CCGATGG ATATTCCGATCAACT, LINC01579p3: ATGGACCGT CGATCCAAGCAT; LY86-AS1p5: ACGGTGCGCA GCATAAGCCA, LY86-AS1p3: GCACTGGTCC GTACAAGCAG.

\section{Statistical analysis}

All of the statistical analysis, including differential expression test and survival analysis, were conducted based on $\mathrm{R}$ version3.2.2 programming software. Differences of expressions of qPCR analysis were tested through two tailed $t$-test with $p$-value $<0.01$ as significance.

\section{CONFLICTS OF INTEREST}

The authors declare that they have no conflicts of interest.

\section{REFERENCES}

1. Johnson DR, O'Neill BP. Glioblastoma survival in the United States before and during the temozolomide era. J Neurooncol. 2012; 107:359-364.

2. Stupp R, Hegi ME, Mason WP, van den Bent MJ, Taphoorn MJ, Janzer RC, Ludwin SK, Allgeier A, Fisher B, Belanger K, Hau P, Brandes AA, Gijtenbeek J, et al. Effects of radiotherapy with concomitant and adjuvant temozolomide versus radiotherapy alone on survival in glioblastoma in a randomised phase III study: 5-year analysis of the EORTCNCIC trial. Lancet Oncol. 2009; 10:459-466.

3. Rich JN, Hans C, Jones B, Iversen ES, McLendon RE, Rasheed BK, Dobra A, Dressman HK, Bigner DD, Nevins JR, West M. Gene expression profiling and genetic markers in glioblastoma survival. Cancer Res. 2005; 65:4051-4058.

4. Okazaki Y, Furuno M, Kasukawa T, Adachi J, Bono H, Kondo S, Nikaido I, Osato N, Saito R, Suzuki H, Yamanaka I, Kiyosawa H, Yagi K, et al. Analysis of the mouse transcriptome based on functional annotation of 60,770 fulllength cDNAs. Nature. 2002; 420:563-573.

5. Gupta RA, Shah N, Wang KC, Kim J, Horlings HM, Wong DJ, Tsai MC, Hung T, Argani P, Rinn JL, Wang Y, Brzoska P, Kong B, et al. Long non-coding RNA HOTAIR reprograms chromatin state to promote cancer metastasis. Nature. 2010; 464:1071-1076.

6. Gibb EA, Brown CJ, Lam WL. The functional role of long non-coding RNA in human carcinomas. Mol Cancer. 2011; 10:38.

7. Mercer TR, Dinger ME, Mattick JS. Long non-coding RNAs: insights into functions. Nat Rev Genet. 2009; 10:155-159.
8. Paraskevopoulou MD, Hatzigeorgiou AG. Analyzing MiRNA-LncRNA Interactions. Methods Mol Biol. 2016; 1402:271-286.

9. Wang KC, Chang HY. Molecular mechanisms of long noncoding RNAs. Mol Cell. 2011; 43:904-914.

10. Liu X, Xiao ZD, Han L, Zhang J, Lee SW, Wang W, Lee H, Zhuang L, Chen J, Lin HK, Wang J, Liang H, Gan B. LncRNA NBR2 engages a metabolic checkpoint by regulating AMPK under energy stress. Nat Cell Biol. 2016; 18:431-442.

11. Inamura K. Major Tumor Suppressor and Oncogenic NonCoding RNAs: Clinical Relevance in Lung Cancer. Cells. 2017; 6 .

12. Ye W, Lv Q, Wong CK, Hu S, Fu C, Hua Z, Cai G, Li G, Yang BB, Zhang Y. The effect of central loops in miRNA:MRE duplexes on the efficiency of miRNAmediated gene regulation. PLoS One. 2008; 3:e1719.

13. Inamura K, Ishikawa Y. MicroRNA In Lung Cancer: Novel Biomarkers and Potential Tools for Treatment. J Clin Med. 2016; 5.

14. Salmena L, Poliseno L, Tay Y, Kats L, Pandolfi PP. A ceRNA hypothesis: the Rosetta Stone of a hidden RNA language? Cell. 2011; 146:353-358.

15. Thomson DW, Dinger ME. Endogenous microRNA sponges: evidence and controversy. Nat Rev Genet. 2016; 17:272-283.

16. Liang WC, Fu WM, Wong $\mathrm{CW}$, Wang Y, Wang WM, Hu GX, Zhang L, Xiao LJ, Wan DC, Zhang JF, Waye MM. The lncRNA H19 promotes epithelial to mesenchymal transition by functioning as miRNA sponges in colorectal cancer. Oncotarget. 2015; 6:22513-22525. https://doi. org/10.18632/oncotarget.4154.

17. Wang Y, Xu Z, Jiang J, Xu C, Kang J, Xiao L, Wu M, Xiong J, Guo X, Liu H. Endogenous miRNA sponge lincRNARoR regulates Oct4, Nanog, and Sox 2 in human embryonic stem cell self-renewal. Dev Cell. 2013; 25:69-80.

18. Zhang Y, Li Y, Wang Q, Zhang X, Wang D, Tang HC, Meng $X$, Ding $X$. Identification of an lncRNAmiRNAmRNA interaction mechanism in breast cancer based on bioinformatic analysis. Mol Med Rep. 2017; 16:5113-5120.

19. Love MI, Huber W, Anders S. Moderated estimation of fold change and dispersion for RNA-seq data with DESeq2. Genome Biol. 2014; 15:550.

20. Gautier L, Cope L, Bolstad BM, Irizarry RA. affy-analysis of Affymetrix GeneChip data at the probe level. Bioinformatics. 2004; 20:307-315.

21. Diboun I, Wernisch L, Orengo CA, Koltzenburg M. Microarray analysis after RNA amplification can detect pronounced differences in gene expression using limma. BMC Genomics. 2006; 7:252.

22. Sherman BT, Huang da W, Tan Q, Guo Y, Bour S, Liu D, Stephens R, Baseler MW, Lane HC, Lempicki RA. DAVID Knowledgebase: a gene-centered database integrating 
heterogeneous gene annotation resources to facilitate highthroughput gene functional analysis. BMC Bioinformatics. 2007; 8:426.

23. Harrow J, Frankish A, Gonzalez JM, Tapanari E, Diekhans M, Kokocinski F, Aken BL, Barrell D, Zadissa A, Searle S, Barnes I, Bignell A, Boychenko V, et al. GENCODE: the reference human genome annotation for The ENCODE Project. Genome Res. 2012; 22:1760-1774.

24. Jeggari A, Marks DS, Larsson E. miRcode: a map of putative microRNA target sites in the long non-coding transcriptome. Bioinformatics. 2012; 28:2062-2063.

25. Chou CH, Chang NW, Shrestha S, Hsu SD, Lin YL, Lee WH, Yang CD, Hong HC, Wei TY, Tu SJ, Tsai TR, Ho SY, Jian TY, et al. miRTarBase 2016: updates to the experimentally validated miRNA-target interactions database. Nucleic Acids Res. 2016; 44(D1):D239-247.

26. Luo W, Brouwer C. Pathview: an R/Bioconductor package for pathway-based data integration and visualization. Bioinformatics. 2013; 29:1830-1831.

27. Hu CC, Gan P, Zhang RY, Xue JX, Ran LK. Identification of prostate cancer LncRNAs by RNA-Seq. Asian Pac J Cancer Prev. 2014; 15:9439-9444.

28. Li Y, Chen J, Zhang J, Wang Z, Shao T, Jiang C, Xu J, Li X. Construction and analysis of lncRNA-lncRNA synergistic networks to reveal clinically relevant lncRNAs in cancer. Oncotarget. 2015; 6:25003-25016. https://doi.org/10.18632/ oncotarget. 4660 .

29. Liu S, Mitra R, Zhao MM, Fan W, Eischen CM, Yin F, Zhao Z. The Potential Roles of Long Noncoding RNAs (lncRNA) in Glioblastoma Development. Mol Cancer Ther. 2016; 15:2977-2986.

30. Brodie S, Lee HK, Jiang W, Cazacu S, Xiang C, Poisson LM, Datta I, Kalkanis S, Ginsberg D, Brodie C. The novel long non-coding RNA TALNEC2, regulates tumor cell growth and the stemness and radiation response of glioma stem cells. Oncotarget. 2017; 8:31785-31801. https://doi. org/10.18632/oncotarget.15991.

31. Zhao T, Xu J, Liu L, Bai J, Wang L, Xiao Y, Li X, Zhang L. Computational identification of epigenetically regulated lncRNAs and their associated genes based on integrating genomic data. FEBS Lett. 2015; 589:521-531.

32. Zhang A, Xu M, Mo YY. Role of the lncRNA-p53 regulatory network in cancer. J Mol Cell Biol. 2014; 6:181-191.

33. Liu JY, Yao J, Li XM, Song YC, Wang XQ, Li YJ, Yan B, Jiang Q. Pathogenic role of 1ncRNA-MALAT1 in endothelial cell dysfunction in diabetes mellitus. Cell Death Dis. 2014; 5:e1506.

34. Kuipers BC, Vulto-van Silfhout AT, Marcelis C, Pfundt R, de Leeuw N, de Vries BB. Two patients with intellectual disability, overlapping facial features, and overlapping deletions in 6p25.1p24.3. Clin Dysmorphol. 2013; 22:18-21.

35. Chi K, Li Y, Ding Q, Xu L, Chen Y, Wang X. High resolution single nucleotide polymorphism array reveals cryptic indicating information about myelodysplastic syndrome-related myeloid malignancies. Leuk Lymphoma. 2015; 56:2969-2972.

36. Liu F, Ji F, Ji Y, Jiang Y, Sun X, Lu Y, Zhang L, Han Y, Liu $\mathrm{X}$. Dissecting the mechanism of colorectal tumorigenesis based on RNA-sequencing data. Exp Mol Pathol. 2015; 98:246-253.

37. Inamura K. Diagnostic and Therapeutic Potential of MicroRNAs in Lung Cancer. Cancers (Basel). 2017; 9.

38. Krishnan K, Steptoe AL, Martin HC, Pattabiraman DR, Nones K, Waddell N, Mariasegaram M, Simpson PT, Lakhani SR, Vlassov A, Grimmond SM, Cloonan N. miR$139-5 p$ is a regulator of metastatic pathways in breast cancer. RNA. 2013; 19:1767-1780.

39. Wong CC, Wong CM, Tung EK, Au SL, Lee JM, Poon RT, Man K, Ng IO. The microRNA miR-139 suppresses metastasis and progression of hepatocellular carcinoma by down-regulating Rho-kinase 2. Gastroenterology. 2011; 140:322-331.

40. Liu R, Yang M, Meng Y, Liao J, Sheng J, Pu Y, Yin L, Kim SJ. Tumor-suppressive function of miR-139-5p in esophageal squamous cell carcinoma. PLoS One. 2013; 8:e77068.

41. De A. Wnt/Ca2+ signaling pathway: a brief overview. Acta Biochim Biophys Sin (Shanghai). 2011; 43:745-756.

42. Xu X, Chen D, Ye B, Zhong F, Chen G. Curcumin induces the apoptosis of non-small cell lung cancer cells through a calcium signaling pathway. Int J Mol Med. 2015; 35:1610-1616.

43. Raynal NJ, Lee JT, Wang Y, Beaudry A, Madireddi P, Garriga J, Malouf GG, Dumont S, Dettman EJ, Gharibyan V, Ahmed S, Chung W, Childers WE, et al. Targeting Calcium Signaling Induces Epigenetic Reactivation of Tumor Suppressor Genes in Cancer. Cancer Res. 2016; 76:1494-1505.

44. Zhang HD, Jiang LH, Sun DW, Li J, Tang JH. MiR-1395p: promising biomarker for cancer. Tumour Biol. 2015; 36:1355-1365.

45. Kim JO, Song DW, Kwon EJ, Hong SE, Song HK, Min CK, Kim DH. miR-185 plays an anti-hypertrophic role in the heart via multiple targets in the calcium-signaling pathways. PLoS One. 2015; 10:e0122509.

46. Yue S, Wang L, Zhang H, Min Y, Lou Y, Sun H, Jiang Y, Zhang W, Liang A, Guo Y, Chen P, Lv G, Wang L. miR-139-5p suppresses cancer cell migration and invasion through targeting ZEB1 and ZEB2 in GBM. Tumour Biol. 2015; 36:6741-6749.

47. Dai S, Wang X, Li X, Cao Y. MicroRNA-139-5p acts as a tumor suppressor by targeting ELTD1 and regulating cell cycle in glioblastoma multiforme. Biochem Biophys Res Commun. 2015; 467:204-210.

48. Bo LJ, Wei B, Li ZH, Wang ZF, Gao Z, Miao Z. Bioinformatics analysis of miRNA expression profile between primary and recurrent glioblastoma. Eur Rev Med Pharmacol Sci. 2015; 19:3579-3586.

49. Xu C, Zhao H, Chen H, Yao Q. CXCR4 in breast cancer: oncogenic role and therapeutic targeting. Drug Des Devel Ther. 2015; 9:4953-4964. 
50. Scala S. Molecular Pathways: Targeting the CXCR4CXCL12 Axis--Untapped Potential in the Tumor Microenvironment. Clin Cancer Res. 2015; 21:4278-4285.

51. Bailey P, Chang DK, Nones K, Johns AL, Patch AM, Gingras MC, Miller DK, Christ AN, Bruxner TJ, Quinn MC, Nourse C, Murtaugh LC, Harliwong I, et al. Genomic analyses identify molecular subtypes of pancreatic cancer. Nature. 2016; 531:47-52.

52. Zhang X, Gu D, Du M, Wang M, Cao C, Shen L, Kuang M, Tan Y, Huo X, Gong W, Xu Z, Chen J, Zhang Z, Tang C. Associations of NR5A2 gene polymorphisms with the clinicopathological characteristics and survival of gastric cancer. Int J Mol Sci. 2014; 15:22902-22917.

53. Shi Y, Chen C, Yu SZ, Liu Q, Rao J, Zhang HR, Xiao HL, Fu TW, Long H, He ZC, Zhou K, Yao XH, Cui YH, et al.
miR-663 Suppresses Oncogenic Function of CXCR4 in Glioblastoma. Clin Cancer Res. 2015; 21:4004-4013.

54. Richardson PJ. CXCR4 and Glioblastoma. Anticancer Agents Med Chem. 2016; 16:59-74.

55. Luo Z, Li Y, Zuo M, Liu C, Yan D, Wang H, Li D. Effect of NR5A2 inhibition on pancreatic cancer stem cell (CSC) properties and epithelial-mesenchymal transition (EMT) markers. Mol Carcinog. 2017; 56:1438-1448.

56. Ueno M, Ohkawa S, Morimoto M, Ishii H, Matsuyama M, Kuruma S, Egawa N, Nakao H, Mori M, Matsuo K, Hosono S, Nojima M, Wakai K, et al. Genome-wide association study-identified SNPs (rs3790844, rs3790843) in the NR5A2 gene and risk of pancreatic cancer in Japanese. Sci Rep. 2015; 5:17018. 